\title{
Nanoscale Bipolar and Complementary Resistive Switching Memory Based on Amorphous Carbon
}

\author{
Yang Chai, Yi Wu, Kuniharu Takei, Hong-Yu Chen, Student Member, IEEE, Shimeng Yu, Student Member, IEEE, \\ Philip C. H. Chan, Fellow, IEEE, Ali Javey, and H.-S. Philip Wong, Fellow, IEEE
}

\begin{abstract}
There has been a strong demand for developing an ultradense and low-power nonvolatile memory technology. In this paper, we present a carbon-based resistive random access memory device with a carbon nanotube (CNT) electrode. An amorphous carbon layer is sandwiched between the fast-diffusing top metal electrode and the bottom CNT electrode, exhibiting a bipolar switching behavior. The use of the CNT electrode can substantially reduce the size of the active device area. We also demonstrate a carbon-based complementary resistive switch (CRS) consisting of two back-to-back connected memory cells, providing a route to reduce the sneak current in the cross-point memory. The bit information of the CRS cell is stored in a high-resistance state, thus reducing the power consumption of the CRS memory cell. This paper provides valuable early data on the effect of electrode size scaling down to nanometer size.
\end{abstract}

Index Terms-Amorphous carbon (a-C), carbon nanotube (CNT), complementary resistive switching, nonvolatile memory, resistive random access memory (RRAM), resistive switching memory.

\section{INTRODUCTION}

B ECAUSE OF the drive toward smaller, faster, and denser nanoelectronics systems, the feature size of the nonvolatile memory continues to scale down toward nanometer size. The conventional Si-based Flash memory is expected to run into its physical limits in the near future [1]. Technology breakthroughs in materials and device concepts are required for next-generation nonvolatile memory. The two-terminal resistive switching memory, where two metal electrodes are separated by a functional insulator, offers the potential for high scalability and ease of integration with current complementary metal-

Manuscript received March 15, 2011; revised May 24, 2011, July 11, 2011, and July 25, 2011; accepted August 1, 2011. Date of publication September 26, 2011; date of current version October 21, 2011. This work was supported in part by the member companies of Stanford Nonvolatile Memory Technology Research Initiative, Stanford Graduate Fellowship, fellowship from the O. G. Villard Engineering Fund at Stanford, the Research Grant Council of Hong Kong Government under Competitive Earmarked Research Grant HKUST 611307, by the Berkeley Sensor and Actuator Center, and by the World Class University Program. The review of this paper was arranged by Editor Y.-H. Shih.

Y. Chai, Y. Wu, H.-Y. Chen, S. Yu, and H.-S. P. Wong are with the Center for Integrated Systems, Department of Electrical Engineering, Stanford University, Stanford, CA 94305 USA (e-mail: hspwong@stanford.edu).

K. Takei and A. Javey are with the Department of Electrical Engineering and Computer Sciences, University of California, Berkeley, CA 94720-1770 USA.

P. C. H. Chan is with the Department of Electronic and Computer Engineering, The Hong Kong University of Science and Technology, Kowloon, Hong Kong, and the Department of Electronic and Information Engineering, The Hong Kong Polytechnic University, Kowloon, Hong Kong.

Color versions of one or more of the figures in this paper are available online at http://ieeexplore.ieee.org.

Digital Object Identifier 10.1109/TED.2011.2164615 oxide-semiconductor technology. To increase the density of the resistive memory, the cross-point architecture, where a bistable material is sandwiched between the parallel bottom electrodes and the orthogonal top electrodes, has been proposed because the memory cell size can be reduced to $4 \mathrm{~F}^{2}(F=$ minimize feature size) and potentially even smaller if the memory cells are stacked in three dimensions [2], [3]. The switching material can be electrically triggered between two resistance states. This resistive switching behavior has been observed in a variety of solid insulating and semiconducting materials, including transition metal oxides (e.g., $\mathrm{NiO}, \mathrm{TiO}_{2}, \mathrm{HfO}_{2}, \mathrm{ZnO}$, and $\mathrm{Al}_{2} \mathrm{O}_{3}$ ) [4]-[7], ferroelectric (e.g., $\mathrm{PbZr}_{0.52} \mathrm{Ti}_{0.48} \mathrm{O}_{3}$ ) [8], ferromagnetic (e.g., $\mathrm{MgO}$ ) [9], perovskite (e.g., $\mathrm{SrZrO}_{3}$ ) [10], chalcogenides (e.g., GeSbTe) [11], organics (e.g., $\mathrm{Alq}_{3}$ ) [12], amorphous silicon (a-Si) [13]-[15], and Si/a-Si core/shell nanowires [16].

Carbon-based materials, including amorphous carbon (a-C) [17]-[26], fullerene [27], graphene oxide [28], [29], carbon/ organic composite [30], and carbon nanotube (CNT) [31], have been shown to exhibit resistive switching behavior for nonvolatile memory application. Amorphous carbon is a noncrystalline carbon allotrope in which a long-range crystalline order is not present. Different switching mechanisms for a-C have been proposed to explain the resistive change behavior, including thermochemical $\mathrm{sp}^{2}$ carbon chain forming/rupture [17], [18], electrochemical metallization [19]-[21], and valence change [22], [23].

To achieve ultrahigh-density cross-point memory, it is necessary to scale down the metal electrodes to nanometer size. However, the fabrication of the nanometer feature size (e.g., 1$2 \mathrm{~nm}$ ) is still challenging for current lithography technologies. At the nanoscale, carrier scattering at the surface leads to a dramatic increase in metal resistivity. This requires researchers to find a way for fabricating nanometer electrodes beyond the limitation of current lithography length scales, and yet maintain the good electrical conductivity at such a small scale. A metallic single-walled CNT, another allotrope in the carbon family, has a nanometer diameter (1-2 nm in this paper), excellent electrical conductivity, and a high current-carrying capacity [32], and it has been used as a nanoscale electrode for resistive switching memory with chalcogenide [33], [34] AgTCNQ [35] and silicon oxide [36], [37]. This bottom-up approach using CNT as the electrode is a promising solution for scaling down the memory device to the nanometer size. The memory device with CNT electrode also enables us to study the resistive switching mechanism of the conduction filament below $5 \mathrm{~nm}$. In this paper, we demonstrate nonvolatile resistive switching for an a-C 

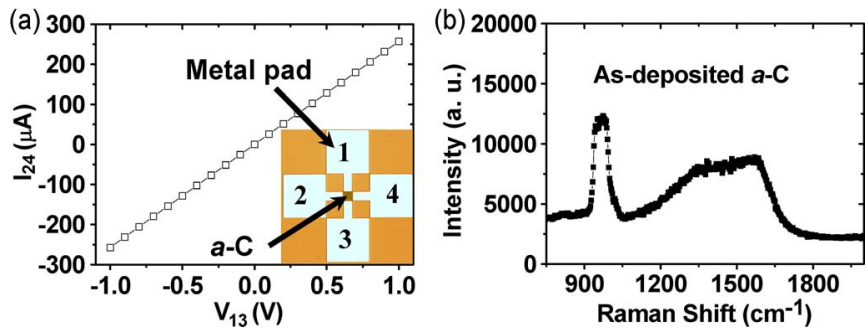

Fig. 1. Characterization of the as-deposited 30-nm-thick a-C film by ebeam evaporation. (a) $I-V$ curve of the a-C film measured by a four-point method. (Inset) Photograph of the four-point measurement structure. (b) Raman spectroscopy of the a-C film, showing a broad peak distribution from 1200 to $1700 \mathrm{~cm}^{-1}$. The strong peak around $1000 \mathrm{~cm}^{-1}$ is the second-order effect from the Si substrate.

layer sandwiched between the top metal electrode and the bottom CNTs. The single-walled CNT, with an average diameter of $1.2 \mathrm{~nm}$, is an ideal nanoscale electrode for ultradense memory cells. We also demonstrate a complementary resistive switch (CRS) [2] for a planar metal/a-C/CNT/a-C/metal device for the first time. This CRS device potentially enables a large passive crossbar array, with the CRS itself serving as an integrated array cell selection device.

\section{Fabrication of the Test Structure}

The chemical bonding of carbon element has $\mathrm{sp}^{1}, \mathrm{sp}^{2}$, and $\mathrm{sp}^{3}$ hybridization configurations. The a-C contains a mixture of $\mathrm{sp}^{3}, \mathrm{sp}^{2}$ and sometimes a small fraction of $\mathrm{sp}^{1}$ sites, and some hydrogen [38]. The $\mathrm{sp}^{2}$ bonding-dominated graphitic carbon has low resistivity close to that of a metal, whereas the $\mathrm{sp}^{3}$ bonding-dominated diamond-like carbon is an insulator with high resistivity. In this paper, we deposited a-C by an electronbeam (e-beam) evaporation technique. A 30-nm-thick a-C was deposited at the pressure of $10^{-5}$ torr. The experimental details are described in the Appendix. To accurately determine the resistivity of the as-deposited a-C film, we used a four-point electrical test structure [see inset in Fig. 1(a)]. Fig. 1(a) shows a typical current-voltage $(I-V)$ curve measured from a 30-nmthick a-C by e-beam evaporation. The resistivity $(121.7 \pm$ $75.7 \Omega \cdot \mu \mathrm{m})$ of the 30 -nm-thick a-C is in the same order with the previous report of a-C by e-beam evaporation and filtered cathodic vacuum arc [22], [39]. Fig. 1(b) shows the Raman spectra of the as-deposited a-C by e-beam evaporation. The $\mathrm{G}$ peak is centered around $1572 \mathrm{~cm}^{-1}$. According to the threestage model by Ferrari and Robertson [40], the G peak position locates in the range between nanocrystalline graphite and a-C. The broad peak distribution from 1200 to $1700 \mathrm{~cm}^{-1}$ indicates the amorphous structure of the carbon film.

Fig. 2(a) shows the process flow for fabricating the metal/ a-C/CNT/metal memory device. Horizontally aligned CNTs were grown on ST-cut single-crystal quartz wafers using Fe catalyst and $\mathrm{CH}_{4}$ as the carbon source. The detailed CNT synthesis process has been described elsewhere [41]. Fig. 2(b) shows a scanning electron microscope (SEM) image of the horizontally aligned CNTs. The average CNT density is $\sim 2 \mathrm{CNT} / \mu \mathrm{m}$ with the number of the CNTs for the typical device with $1-\mu \mathrm{m}$ width varying from $1-3$. The length of the CNT is $200 \mu \mathrm{m}$ in this paper, making the use of CNT for long (a) CNT transfer a-C deposition Metal deposition CNT etching

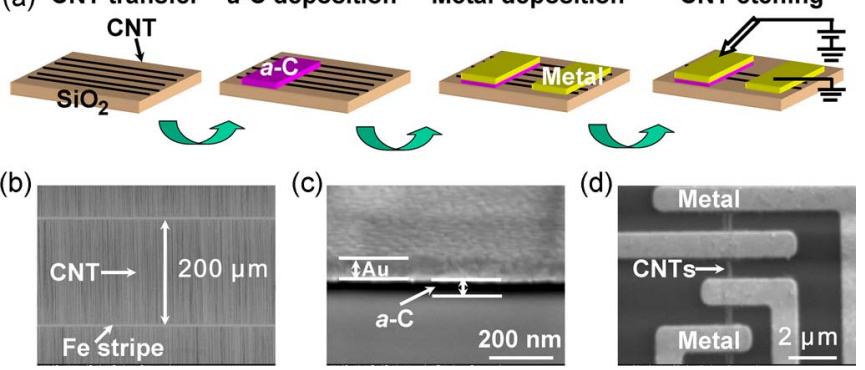

Fig. 2. Fabrication of the metal/a-C/CNT/metal memory device. (a) Process flow for fabricating the memory structure. (b) SEM image of the horizontally aligned CNTs with 200- $\mu$ m length. (c) Cross-sectional SEM image of the carbon memory cell, showing the metal electrode on top of the a-C layer. (d) Top-view SEM image of the carbon memory cell, showing that the CNT acts as the bottom electrode of the memory cell.

bit and word lines possible. Earlier work showed the aligned CNT can be $\sim 1 \mathrm{~mm}$ long [41]. The horizontally aligned CNTs were then transferred to a $\mathrm{Si} / \mathrm{SiO}_{2}$ substrate using a $100-\mathrm{nm}$ thick Au film and a thermal release adhesive tape [41]. The a-C pattern was defined by a standard photolithography process. A 30-nm-thick a-C layer was deposited on top of the transferred CNT by e-beam evaporation, followed by a liftoff process. The top metal electrodes were patterned by a second e-beam evaporation process and liftoff. In this paper, we used a fast-diffusing metal, e.g., Ag or Au. Thus, the a-C layer was sandwiched between the top metal electrode and the bottom CNTs. Fig. 2(c) shows a cross-sectional SEM image of the a-C memory cell. The unwanted CNTs were etched away by the oxygen plasma, leaving the CNT only in the active region. Fig. 2(d) shows a top-view SEM image of one memory cell. The CNTs act as the bottom electrode in the memory cell, and they are orthogonal to the top metal electrode.

\section{RESUlts}

The capping metal on top of a-C plays an important role in determining the switching behavior. Unipolar switching has been reported for the a-C layer with an inert metal electrode such as $\mathrm{W}$ and $\mathrm{Cr}$ [17], [18]. The unipolar switching mechanism of a-C has been explained as the formation/rupture of the $\mathrm{sp}^{2}$ carbon chain in the $\mathrm{sp}^{3}$ carbon matrix, in which carbon is the monoatom for both the conductive filament and the insulating matrix. Devices with a fast-diffusing metal such as $\mathrm{Cu}, \mathrm{Ag}$, and $\mathrm{Au}$ on top of a-C have been reported to exhibit the bipolar resistive switching behavior [19]-[21]. The bipolar resistive switching behavior is the result of electrochemical metallization of the conductive filaments, similar to the conductive-bridge resistive memory [13]-[15], [19]-[21]. Bipolar switching is more controllable than unipolar switching as the SET and the RESET processes occur at opposite voltage polarities. In this paper, we used a fast-diffusing metal ( $\mathrm{Ag}$ or $\mathrm{Au}$ ) as the top metal electrode and CNTs as the nanoscale bottom electrode. DC voltage sweeps were applied at the top metal electrode with the bottom CNT electrode grounded [see Fig. 2(a)]. The initial state of the memory device exhibits the high-resistance state (HRS) around $5 \mathrm{M} \Omega$. A positive dc sweep was used to switch the memory cell to the low-resistance state (LRS). The dc 

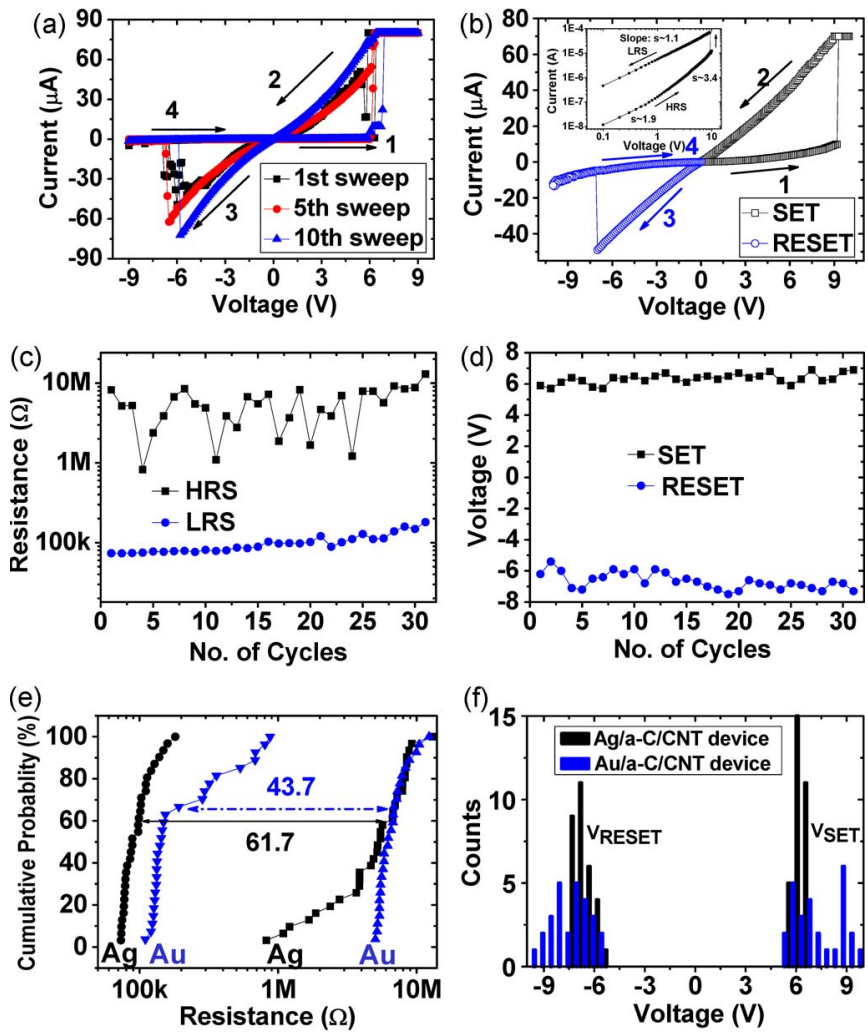

Fig. 3. Electrical characterization of the carbon-based bipolar memory. (a) $I-V$ curves of a $\mathrm{Ag} / \mathrm{a}-\mathrm{C} / \mathrm{CNT} / \mathrm{Ag}$ memory cell. The dc sweep was performed from $0 \rightarrow 9 \mathrm{~V} \rightarrow 0 \rightarrow-9 \mathrm{~V} \rightarrow 0$. The current compliance is $80 \mu \mathrm{A}$. (b) $I-V$ curve of a Au/a-C/CNT/Au memory cell. The dc sweep was performed from $0 \rightarrow 10 \mathrm{~V} \rightarrow 0 \rightarrow-10 \mathrm{~V} \rightarrow 0$. The current compliance is $70 \mu \mathrm{A}$. Inset of (b) shows the $I-V$ curve of the Au/a-C/CNT/Au memory cell in the $\log -\log$ scale. Evolution of (c) HRS/LRS, where the resistance state is read at $1 \mathrm{~V}$, and (d) $V_{\mathrm{SET}} / V_{\mathrm{RESET}}$ of a Ag/a-C/CNT/Ag cell. Statistical distribution of (e) HRS/LRS and (f) $V_{\mathrm{SET}} / V_{\mathrm{RESET}}$ of Ag/a-C/CNT/Ag and Au/a-C/ $\mathrm{CNT} / \mathrm{Au}$ cells during endurance testing.

voltage was swept in the sequence of $0 \rightarrow V \rightarrow 0 \rightarrow-V \rightarrow 0$ with current compliance below $100 \mu \mathrm{A}$. Fig. 3(a) shows the first, fifth, and tenth sweeps of a Ag/a-C/CNT/Ag cell. SET switching was swept with current compliance $(80 \mu \mathrm{A}$ in this curve) to prevent the device from suffering permanent breakdown. The resistance state of the memory cell was switched from HRS to LRS about $6.2 \mathrm{~V}$ and switched back to HRS only by applying a negative dc voltage, exhibiting the typical bipolar switching behavior. The resistance OFF/ON ratio at $5 \mathrm{~V}$ is around 80. The Au/a-C/CNT/Au device [see Fig. 3(b)] shows similar resistive switching behaviors to the $\mathrm{Ag} / \mathrm{a}-\mathrm{C} / \mathrm{CNT} / \mathrm{Ag}$ device. Inset in Fig. 3(b) shows the log-log scale $I-V$ curve of the memory cell. The current-voltage relationship indicates the different conduction mechanisms in HRS and LRS. In our device configuration, the bottom CNT electrode is chemically inert. No atomic migration will happen in the CNT in the normal device operation region due to the high current-carrying capacity $\left(>10^{9} \mathrm{~A} / \mathrm{cm}^{2}\right)$ of the CNT [32]. Resistive switching is most likely to be caused by the precipitates from the top diffusing metal electrode to the a-C matrix [19]-[21]. The $I-V$ curve of the LRS of a Au/a-C/CNT/Au cell shows an ohmiclike behavior with a slope of 1.1. This is believed to be from the formation of a $\mathrm{Au}$ or Ag conduction filament during the SET process [19]-[21]. The charge transport in HRS is in agreement
TABLE I

Parameters of the CARbon-BASEd Memory Cells With DifFERENT ACTIVE DEVICE AREAS

\begin{tabular}{|c|c|c|c|c|}
\hline & This work & \multicolumn{2}{|c|}{ Choi'09 } & Zhuge'10 \\
\hline Device & $\mathrm{Ag} / \mathrm{a}-\mathrm{C} / \mathrm{CNT}$ & \multirow{2}{*}{\multicolumn{2}{|c|}{$\begin{array}{l}\mathrm{Pt} / \mathrm{Cu}-\mathrm{C} / \mathrm{Pt} \\
\text { sputtering }\end{array}$}} & $\mathrm{Cu} / \mathrm{a}-\mathrm{C} / \mathrm{Pt}$ \\
\hline C deposition & e-beam & & & ion-beam \\
\hline Area $\left(\mu \mathrm{m}^{2}\right)$ & 0.001 & 0.01 & 25 & $10^{4}$ \\
\hline Reset I $(\mu \mathrm{A})$ & $40-75$ & 206.4 & 6985 & $\sim 12000$ \\
\hline Reset V (V) & $5.4-7.5$ & 1.1 & N/A & $0.8-1.2$ \\
\hline ON/OFF & $40-200$ & $\sim 100$ & $\sim 2.24$ & $>100$ \\
\hline Retention & $>10^{6} \mathrm{~s}$ & $N / A$ & N/A, & $>10^{5} \mathrm{~s}$ \\
\hline
\end{tabular}

with a trap-controlled space-charge-limited current mechanism [19], [22]. The ON and OFF states of the memory cells retained for at least two weeks at room temperature in air. No electrical power was needed to maintain the resistance states, indicating the nonvolatile property of the memory cell.

The use of the nanoscale CNT electrode enables us to study the effect of the scaling of the active area on power consumption. The active area of the memory device can be estimated by the width of the top metal electrode and the diameter of the bottom CNT, around $1 \mu \mathrm{m} \times 0.001 \mu \mathrm{m}=0.001 \mu \mathrm{m}^{2}$. Table I documents the comparisons of major memory parameters between our devices and the other a-C-based memory cells [19][21]. Although the memory devices were fabricated by different processes (different carbon deposition methods and different top metal electrodes), all of them exhibit a similar bipolar switching behavior. We listed the parameters of the devices with different active areas in Table I. The RESET current is defined as the maximum current that is attained before an abrupt decrease in resistance. The dramatic decrease in the RESET current is noticeably observed when the active device area is reduced, whereas the resistance ON/OFF ratio and the retention time of our nanoscale device remain comparable to those of the larger device. These results suggest that the downward scaling of the active device area can reduce the RESET current of the a-C-based memory device [19]-[21]. The reduction of the active device area using the CNT electrode confines the conduction filament more locally and reduces the RESET current.

The cycling measurements were repeated by the dc sweep. Fig. 3(c) shows the resistance evolution of HRS and LRS of a $\mathrm{Ag} / \mathrm{a}-\mathrm{C} / \mathrm{CNT} / \mathrm{Ag}$ device during the dc sweep cycling. Resistance switching is reproducible, and the memory cell successfully operates over 31 times. Fig. 3(d) shows the evolution of $V_{\mathrm{SET}}$ and $V_{\mathrm{RESET}}$ during endurance testing. The $V_{\mathrm{SET}}$ and $V_{\text {RESET }}$ of Ag/a-C/CNT/Ag are higher than those of the metal/ a-C/metal structure [19]-[21], probably related to the bottom $\mathrm{CNT} /$ metal contact. The contact area at the nanoscale electrode is very small, resulting in current crowding. The actual contact resistance of CNT is much larger than the ideal quantum resistance limit. There is a large voltage drop at the interface between CNT and surrounding materials (CNT/metal and CNT/ a-C). Fig. 3(e) and (f) shows the statistical distribution of the HRS/LRS resistance and the SET/RESET voltage for Ag/a-C/ $\mathrm{CNT} / \mathrm{Ag}$ and $\mathrm{Au} / \mathrm{a}-\mathrm{C} / \mathrm{CNT} / \mathrm{Au}$ memory cells during endurance 
testing. In LRS, the overall resistance of the cell is hundreds of kiloohms, close to that of the CNT device directly contacted with metal. In HRS, the presence of some $\mathrm{sp}^{3}$ carbon results in the overall resistance of the cell on the order of megaohms. Both the HRS and LRS resistance values of the memory cells show a large variation during endurance testing. Our results are in contrast to the better uniformity for a nanoscale memory device (electrode diameter $\Phi=50 \mathrm{~nm}$ ) [42]. This difference possibly results from the effect of further electrode downscaling. For a large memory device, (e.g., $\Phi>1 \mu \mathrm{m}$ ), the conductive filaments' formation process is determined by the competition among many possible filamentary paths [19], resulting in a large variation of the resistance states during endurance testing [42]. For a nanoscale device, (e.g., $\Phi=50 \mathrm{~nm}$ ) [42], the conduction filament is confined in a nanoscale location. Limited possible filamentary paths are competitive for the formation of the filament. As a result, the nanoscale memory device during endurance testing exhibits better uniformity. For our nanoscale memory device, (the average diameter of the CNT electrode is $\sim 1.2 \mathrm{~nm}$ ), the CNT electrode is comparable to or even smaller than the size of the conductive filament. In the ON state, the small contact area between the bottom CNT and the conductive filament makes electrical coupling extremely difficult. The dominant factor becomes the interface between the nanometer electrode and the conduction filament instead of the formation of different filamentary conduction paths. In each switching cycle, the contact configuration at nanoscale is quite different in the small contact area. It is reasonable to expect the resistance states and $V_{\mathrm{SET}} / V_{\mathrm{RESET}}$ to have a large variation in our nanometer memory device.

We have successfully demonstrated the nanoscale resistive memory based on the structure of metal/a-C/CNT/metal. To further increase the density of the memory, cross-point memory is preferred. The sneak leakage current is an inherent disadvantage of cross-point memory [2]. A selection diode integrated with the memory cell is often proposed to solve the sneak current path problem [3]. Recently, CRSs have been proposed for solving the sneak leakage current in cross-point memory, in which two bipolar resistive memory cells are connected back to back [2]. In our structure, the two series memory cells were simultaneously fabricated, alleviating the impact from fabricating the top cell on the bottom cell. We fabricated the carbonbased lateral CRS structure with two bipolar metal/a-C/CNT/ metal cells connected back to back by a common CNT as a metal/a-C/CNT/a-C/metal device. The fabrication process has been schematically described in Fig. 4(a). The difference between the metal/a-C/CNT/metal memory and metal/a-C/CNT/ $\mathrm{a}-\mathrm{C} /$ metal memory devices lies in the pattern of the a-C layer. For the metal/a-C/CNT/metal memory device, a-C only deposited on top of one end of the CNT; for the metal/a-C/CNT/ a-C/metal device, both ends of the CNT are contacted with a-C/metal.

Inset in Fig. 4(b) shows the resistance measurement of a $\mathrm{Ag} / \mathrm{a}-\mathrm{C} / \mathrm{CNT} / \mathrm{a}-\mathrm{C} / \mathrm{Ag}$ cell during the initialization process. The as-fabricated two memory cells are both at HRS initially. This HRS/HRS in CRS only exists in the pristine device. When a positive voltage sweep is applied to the CRS cell, one (cell 1) of the two memory cells is triggered to LRS. The decreasing
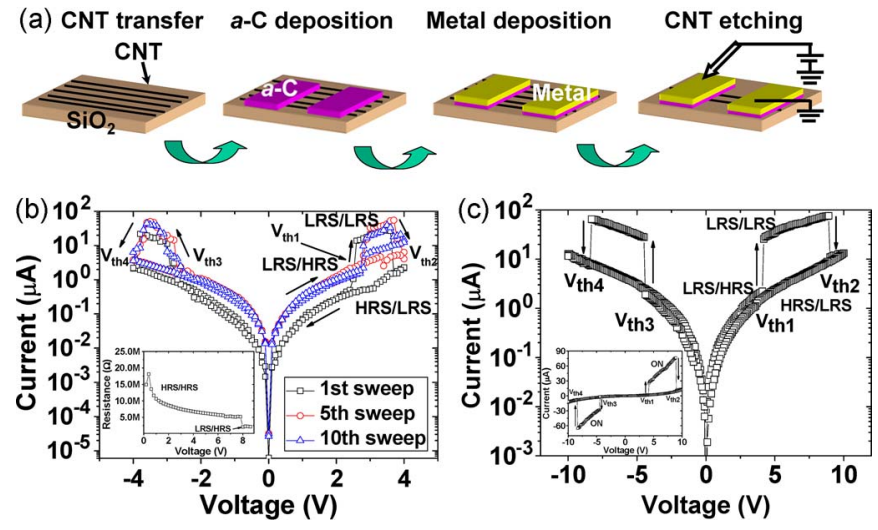

Fig. 4. $I-V$ curves of the CRS memory cell. (a) Schematic of the process flow for fabricating the metal/a-C/CNT/a-C/metal CRS cell. (b) $I-V$ curves of a Ag/a-C/CNT/a-C/Ag CRS cell. The dc sweep was performed from $0 \rightarrow$ $4 \mathrm{~V} \rightarrow 0 \rightarrow-4 \mathrm{~V} \rightarrow 0$. Inset of (b) shows the resistance measurement of the initialization process of the CRS cell. The resistance state of the CRS cell is converted from initial HRS/HRS to LRS/HRS. (c) Semilog $I-V$ curve of a Au/a-C/CNT/a-C/Au CRS cell. The dc sweep was performed from $0 \rightarrow$ $10 \mathrm{~V} \rightarrow 0 \rightarrow-10 \mathrm{~V} \rightarrow 0$. Inset of (c) shows the linear scale $I-V$ curve of the $\mathrm{Au} / \mathrm{a}-\mathrm{C} / \mathrm{CNT} / \mathrm{a}-\mathrm{C} / \mathrm{Au}$ cell.

resistance is observed in the inset in Fig. 4(b). Because the polarity of the SET voltage in the other cell (cell 2) is opposite, it remains in the HRS and acts as a voltage divider. When we continue to sweep the voltage from zero to $-V_{\text {th } 3}$ (beyond the SET voltage of cell 2), both cells are in LRS. When the negative voltage is increased to $-V_{\text {th4 } 4}$, cell 1 is RESET to its HRS. Fig. 4(b) shows the typical $I-V$ curves of a Ag/a-C/CNT/a-C/ Ag CRS cell, which is a superimposed $I-V$ characteristic of the two bipolar memory cells connected back to back. The four distinct threshold voltages enable us to define the CRS cell with four different states [i.e., "ON" (LRS/LRS), "OFF" (HRS/ HRS), "0" (LRS/HRS), and "1" (HRS/LRS)] [2]. The bit information is stored in the two back-to-back memory cells, whereas the overall resistance of the CRS remains dominated by the HRS, i.e., $R_{\text {HRS }}$. The CRS cell exhibits overall high resistance when storing the bit information, thus effectively reducing the sneak current to the unselected cell. The use of two distinct HRSs to store the bit information also reduces static power consumption [2], while at the same time, this scheme is subject to read/write noise margin constraints [43]. The Ag/a-C/CNT/ a-C/Ag CRS cell in Fig. 4(b) shows poor endurance performance. The cell failed only after 11 cycles. In addition to the reasons similar to the $\mathrm{Ag} / \mathrm{a}-\mathrm{C} / \mathrm{CNT} / \mathrm{Ag}$ cell, the poor endurance of the cell is also related to the CRS structure itself. The two cells in the CRS structure are not identical due to device variations. The undesired RESET process may happen in one cell before the desired SET in the other cell. Fig. 4(c) shows the $I-V$ curve from a Au/a-C/CNT/a-C/Au cell. It is found to have a larger ON/OFF ratio and a larger switching voltage than those of the $\mathrm{Ag} / \mathrm{a}-\mathrm{C} / \mathrm{CNT} / \mathrm{a}-\mathrm{C} / \mathrm{Ag}$ cell. This indicates that the top metal electrode plays an important role on the performance of the memory cell.

\section{CONCLUSION}

In summary, we have successfully demonstrated a new carbon-based resistive random access memory device. The use 
of the CNT as an electrode leads to the ultimately scaled crosspoint area. The operation current is greatly reduced, providing the advantages, which lead themselves to a low-power device. We have also shown a carbon-based CRS memory cell for the first time. This new structure has the potential for use in dense cross-point memory without the cell selection devices.

\section{APPENDIX}

The resistivity of carbon film is closely related to the deposition process. In this paper, we used an Edwards EB3 electron beam evaporator, and we used a graphite target as a carbon source. We conduct the deposition process at the pressure of $10^{-5}$ torr. The graphite has a very high melting point. To make sure that the carbon is evaporated, we used the beam current $80-100 \mathrm{~mA}$ at the voltage of $5.6 \mathrm{kV}$. The through distance between the target and the substrate is short $(\sim 30 \mathrm{~cm})$. The strong radiation from the carbon target heats the substrate. In this paper, the deposition process is actually not a roomtemperature process. The thermal couple close to the sample holder displays over $135^{\circ} \mathrm{C}$. The actual substrate temperature is even higher than this value. Deposition of a 30-nm-thick carbon film takes $12-15 \mathrm{~min}$. This process at elevated temperature is similar to an in situ anneal, it and helps reduce the resistivity of carbon film [38].

\section{REFERENCES}

[1] R. Bez and A. Pirovano, "Non-volatile memory technologies: Emerging concepts and new materials," Mater. Sci. in Semiconductor Processing, vol. 7, no. 4-6, pp. 349-355, Nov. 2004.

[2] E. Linn, R. Rosezin, C. Kugeler, and R. Waser, "Complementary resistive switches for passive nanocrossbar memories," Nat. Mater., vol. 9, pp. 403-406, Apr. 2010.

[3] J. Liang and H.-S. P. Wong, "Cross-point memory array without cell selectors-Device characteristics and data storage pattern dependencies," IEEE Trans. Elec. Dev., vol. 57, no. 10, pp. 2531-2538, Oct. 2010.

[4] N. Xu, L. F. Liu, X. Sun, X. Y. Liu, D. D. Han, Y. Wang, R. Q. Han, J. F. Kang, and B. Yu, "Characteristics and mechanism of conduction/set process in TiN/ZnO/Pt resistance switching random-access memories," Appl. Phys. Lett., vol. 92, p. 232112, Jun. 2008.

[5] W. Y. Chang, Y. C. Lai, T. B. Wu, S. F. Wang, F. Chen, and M. J. Tsai, "Unipolar resistive switching characteristics of $\mathrm{ZnO}$ thin films for nonvolatile memory applications," Appl. Phys. Lett., vol. 92, p. 022110, Jan. 2008 .

[6] B. J. Choi, D. S. Jeong, S. K. Kim, C. Rohde, S. Choi, J. H. Oh, H. J. Kim, C. S. Hwang, K. Szot, R. Waser, B. Reichenberg, and S. Tiedke, "Resistive switching mechanism of $\mathrm{TiO}_{2}$ thin films grown by atomic-layer deposition," J. Appl. Phys., vol. 98, p. 033715, Aug. 2005.

[7] Y. C. Yang, F. Pan, Q. Liu, M. Liu, and F. Zeng, "Fully room-temperaturefabricated nonvolatile resistive memory for ultrafast and high-density memory application," Nano Lett., vol. 9, no. 4, pp. 1636-1643, Mar. 2009.

[8] J. R. Contreras, H. Kohlstedt, U. Poppe, R. Waser, C. Buchal, and N. A. Pertsev, "Resistive switching in metal-ferroelectric-metal junctions," Appl. Phys. Lett., vol. 83, no. 22, pp. 4595-4597, Dec. 2003.

[9] S. Yuasa, T. Nagahama, A. Fukushima, Y. Suzuki, and K. Ando, "Giant room-temperature magnetoresistance in single-crystal $\mathrm{Fe} / \mathrm{MgO} / \mathrm{Fe}$ magnetic tunnel junctions," Nat. Mater., vol. 3, no. 12, pp. 868-871, Oct. 2004.

[10] J. W. Park, M. K. Yang, and J. K. Lee, "Electrode dependence of bipolar resistive switching in SrZrO3: Cr perovskite film-based memory devices," Electrochemical and Solid State Lett., vol. 11, no. 8, pp. H226-H229, Aug. 2008.

[11] A. Pirovano, A. L. Lacaita, F. Pellizzer, S. A. Kostylev, A. Benvenuti, and R. Bez, "Low-field amorphous state resistance and threshold voltage drift in chalcogenide materials," IEEE Trans. Elec. Dev., vol. 51, no. 5, pp. 714-719, May 2004.
[12] L. D. Bozano, B. W. Kean, V. R. Deline, J. R. Salem, and J. C. Scott, "Mechanism for bistability in organic memory elements," Appl. Phys. Lett., vol. 84, no. 4, pp. 607-609, Jan. 2004.

[13] S. H. Jo, K.-H. Kim, and W. Lu, "High-density crossbar arrays based on a Si memristive system," Nano Lett., vol. 9, no. 2, pp. 870-874, Feb. 2009.

[14] S. H. Jo and W. Lu, "CMOS compatible nanoscale nonvolatile resistance switching memory," Nano Lett., vol. 8, pp. 392-397, Jan. 2008.

[15] S. H. Jo, K.-H. Kim, and W. Lu, "Programmable resistance switching in nanoscale two-terminal devices," Nano Lett., vol. 9, no. 1, pp. 496-500, Jan. 2009.

[16] Y. Dong, G. Yu, M. C. McAlpine, W. Lu, and C. M. Lieber, "Si/a-Si core/shell nanowires as nonvolatile crossbar switches," Nano Lett., vol. 8, no. 2, pp. 386-391, Jan. 2008.

[17] F. Kreupl, R. Bruchhaus, P. Majewski, J. B. Philipp, R. Symanczyk, T. Happ, C. Arndt, M. Vogt, R. Zimmermann, A. Buerke, A. P. Graham, and M. Kund, "Carbon based resistive memory," IEDM Tech. Dig., pp. 521-524, Dec. 2008.

[18] A. Sinitskii and J. M. Tour, "Lithographic graphitic memories," ACS Nano, vol. 3, no. 9, pp. 2760-2766, Aug. 2009.

[19] F. Zhuge, W. Dai, C. L. He, A. Y. Wang, Y. W. Liu, M. Li, Y. H. Wu, P. Cui, and R. W. Li, "Nonvolatile resistive switching memory based on amorphous carbon," Appl. Phys. Lett., vol. 96, no. 16, p. 163505, Apr. 2010.

[20] H. Choi, M. Pyun, T. W. Kim, M. Hasan, R. Dong, J. Lee, J. B. Park, J. Yoon, D. J. Seong, T. Lee, and H. Hwang, "Nanoscale resistive switching of a copper-carbon-mixed layer for nonvolatile memory applications," IEEE Electron Dev. Lett., vol. 30, no. 3, pp. 302-304, Mar. 2009.

[21] M. Pyun, H. Choi, J. B. Park, D. Lee, M. Hasan, R. Dong, S. J. Jung, J. Lee, D. J. Seong, J. Yoon, and H. Hwang, "Electrical and reliability characteristics of copper-doped carbon (CuC) based resistive switching devices for nonvolatile memory applications," Appl. Phys. Lett., vol. 93, no. 21, p. 212907 , Nov. 2008.

[22] E. G. Gerstner and D. R. McKenzie, "Nonvolatile memory effects in nitrogen doped tetrahedral amorphous carbon thin films," J. Appl. Phys., vol. 84, no. 10, pp. 5647-5651, Oct. 1998.

[23] E. G. Gerstner and D. R. McKenzie, "Cycling effects in nitrogen doped tetrahedral amorphous carbon non-volatile memory cells," Solid Stat. Electronics, vol. 44, no. 9, pp. 1641-1645, Sep. 2000.

[24] B. Standley, W. Bao, H. Zhang, J. Bruck, C. N. Lau, and M. Bockrath "Graphene-based atomic-scale switches," Nano. Lett., vol. 8, no. 10, pp. 3345-3349, Aug. 2008.

[25] Y. Chai, Y. Wu, K. Takei, H. Y. Chen, S. M. Yu, P. C. H. Chan, A. Javey, and H.-S. P. Wong, "Resistive switching of carbon-based RRAM with CNT electrodes for ultra-dense memory," in IEDM Technical Digest, Dec. 2010, pp. 214-217.

[26] D. Fu, D. Xie, T. Feng, C. Zhang, J. Niu, H. Qian, and L. Liu, "Unipolar resistive switching properties of diamondlike carbon-based RRAM devices," IEEE Electr. Dev. Lett., vol. 32, no. 6, pp. 803-805, Jun. 2011

[27] D. A. Corley, T. He, and J. M. Tour, "Two-terminal molecular memories from solution-deposited $\mathrm{C}_{60}$ films in vertical silicon nanogaps," ACS Nano, vol. 4, no. 4, pp. 1879-1888, Mar. 2010.

[28] H. Y. Jeong, J. Y. Kim, J. W. Kim, J. O. Hwang, J. E. Kim, J. Y. Lee, T. H. Yoon, B. J. Cho, S. O. Kim, R. S. Ruoff, and S. Y. Choi, "Graphene oxide thin films for flexible nonvolatile memory applications," Nano Lett., vol. 10 , no. 11 , pp. $4381-4386$, Oct. 2010.

[29] C. L. He, F. Zhuge, X. F. Zhou, M. Li, G. C. Zhou, Y. W. Liu, J. Z. Wang, B. Chen, W. J. Su, Z. P. Liu, Y. H. Wu, P. Cui, and R. W. Li, "Nonvolatile resistive switching in graphene oxide thin films," Appl. Phys. Lett., vol. 95, no. 23, p. 232101, Dec. 2009.

[30] D. Wei, J. K. Baral, R. Osterbacka, and A. Ivaska, "Memory effect in an ionic liquid matrix containing single-walled carbon nanotubes and polystyrene," Nanotechnology, vol. 19, no. 5, p. 055203, Jan. 2008.

[31] J. Yao, Z. Jin, L. Zhong, D. Natelson, and J. M. Tour, "Two-terminal nonvolatile memories based on single-walled carbon nanotubes," ACS Nano, vol. 3, no. 12, pp. 4122-4126, Nov. 2009.

[32] Y. Chai and P. C. H. Chan, "High electromigration-resistant copper/carbon nanotube composite for interconnect application," in IEDM Tech. Dig., Dec. 2008, pp. 607-610.

[33] F. Xiong, A. Liao, and E. Pop, "Inducing chalcogenide phase change with ultra-narrow carbon nanotube heaters," Appl. Phys. Lett., vol. 95, no. 24, p. 243103 , Dec. 2009.

[34] F. Xiong, A. Liao, D. Estrada, and E. Pop, "Low power switching of phase-change materials with carbon nanotube electrodes," Science, vol. 332, pp. 568-570, Apr. 2011.

[35] W. Zhou, L. Ren, F. Lin, L. Jiao, T. Xue, X. Xian, and Z. Liu, "An electrical switch based on Ag-tetracyanoquinodimethane sandwiched by 
crossed carbon nanotube electrodes," Appl. Phys. Lett., vol. 93, no. 12, p. 123115 , Sep. 2008 .

[36] J. Yao, L. Zhong, Z. Zhang, T. He, Z. Jin, P. J. Wheeler, D. Natelson, and J. M. Tour, "Resistive Switching in Nanogap systems on $\mathrm{SiO} 2$ substrates," Small, vol. 5, pp. 2910-2915, 2009.

[37] J. Yao, Z. Sun, L. Zhong, D. Natelson, and J. M. Tour, "Resistive switches and memories from Silicon Oxide," Nano Lett., vol. 10, pp. 4105-4110, 2010.

[38] J. Robertson, "Electronic and atomic structure of diamond-like carbon," Semiconductor Sci. and Tech., vol. 18, no. 3, pp. S12-S19, Feb. 2003.

[39] J. Rao, K. J. Lawson, and J. R. Nicholls, "The characterization of e-beam evaporated and magnetron sputtered carbon films," Surface \& Coatings Technology, vol. 197, pp. 154-160, 2005.

[40] C. Ferrari and J. Robertson, "Interpretation of Raman spectra of disordered and amorphous carbon," Phys. Rev. B, vol. 61, no. 20, pp. 14095-14107, 2000.

[41] N. Patil, A. Lin, E. R. Myers, K. Ryu, A. Badmaev, C. Zhou, H.-S. P. Wong, and S. Mitra, "Wafer-scale growth and transfer of aligned single-walled carbon nanotubes," IEEE Trans. Nano., vol. 8, no. 4, pp. 498-504, Jul. 2009.

[42] D. J. Seong, J. Park, N. Lee, M. Hasan, S. Jung, H. Choi, J. Lee, M. Jo, W. Lee, S. Park, S. Kim, Y. H. Jang, Y. Lee, M. Sung, D. Kil, Y. Hwang, S. Chung, S. Hong, J. Roh, and H. Hwang, "Effect of Oxygen migration and interface engineering on resistance switching behavior of reactive metal/Polycrystalline $\operatorname{Pr} 0.7 \mathrm{Ca} 0.3 \mathrm{MnO} 3$ device for nonvolatile memory application," in IEDM Tech. Dig., Dec. 2009, pp. 101-104.

[43] S. Yu, J. Liang, Y. Wu, and H.-S. P. Wong, "Read/write schemes analysis for the novel complementary resistive switches in passive crossbar memory arrays," Nanotechnology, vol. 21, p. 465202, 2010.

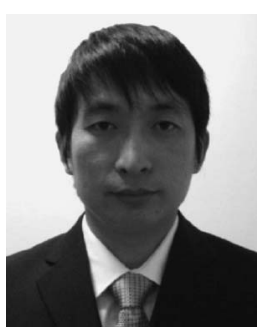

Yang Chai received the Ph.D. degree from The Hong Kong University of Science and Technology, Kowloon, Hong Kong, in 2009.

$\mathrm{He}$ is currently working on the fabrication of carbon-related materials and carbon-based devices, including testing and characterization of resulting prototypes in the Department of Electrical Engineering, Stanford University, CA.

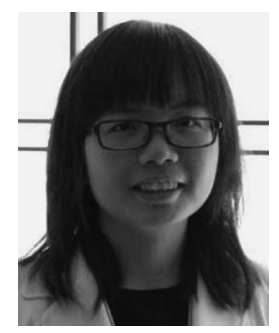

Yi Wu received the B.S. degree in 2008 from Peking University, Beijing, China, and the M.S. degree in 2010 from Stanford University, Stanford, CA, where she is currently working toward the Ph.D. degree in the Department of Electrical Engineering.

She was a Research Intern with Industrial Technology Research Institute, Hsinchu, Taiwan, in 2010 and a Device Intern with IBM Corporation, East Fishkill, NY, in 2011. She is also the author of more than 10 technical papers, including several papers in Symposia of VLSI Technology, IEDM, IEEE Electron Device of LeTters, etc. She is working on metal-oxidebased resistance switching memory with focus on thin-film property study and memory structure innovations.

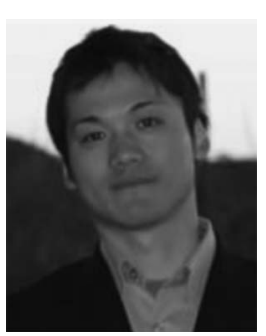

Kuniharu Takei received the B.S., M.S., and Ph.D. degrees from Toyohashi University of Technology, Toyohashi, Japan, in 2003, 2006, and 2009, respectively, all in electrical engineering.

During his Ph.D. studies, he focused on complementary metal-oxide-semiconductor/microelectromechanical system integration for neural electronic interfaces such as spike signal recordings and drug deliveries. He has been a Postdoctoral Fellow with the University of California, Berkeley, since 2009. His research interests in Berkeley are nanomateria integrations for high-performance electronics on any substrates such as conventional $\mathrm{Si}$ and flexible substrates.

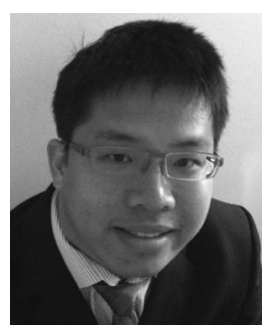

Hong-Yu (Henry) Chen (S'10) received the B.S. degree in electrical engineering in 2007 from $\mathrm{Na}$ tional Tsing Hua University, Hsinchu, Taiwan, and the M.S. degree in electrical engineering in 2011 from Stanford University, Stanford, CA, where he is currently working toward the Ph.D. degree in the Center for Integrated Systems.

In the summer of 2011, he was an Intern with Applied Materials, Sunnyvale, CA, where he worked on high- $\kappa$ metal gate modeling. His research interests in carbon nanotubes include material synthesis, novel transistor device structures, very large scale integration fabrication and processing, and applications in phase-change memory.

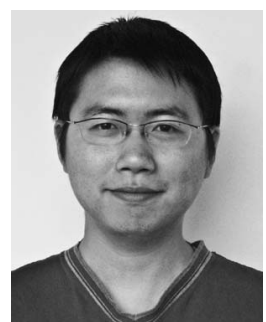

Shimeng Yu (S'10) received the B.S. degree in 2009 from Peking University, Beijing, China, and the M.S. degree in 2011 from Stanford University, Stanford, $\mathrm{CA}$, where he is currently working toward the Ph.D. degree.

He has authored or coauthored more than 30 papers related to his research interests, including several papers in IEDM, Symposia of VLSI Technology, IEEE TRANSACTIONS ON ELECTRON DeVices, IEEE EleCtron DEVICE LETTERS, Applied Physics Letters, Nanotechnology, etc. His previous research activities include simulation of parameter fluctuation in nanoscale transistors and static random access memory cells. He is currently working on the fabrication, characterization, and modeling of emerging resistive switching memory devices and their applications for neuromorphic computation system.

Dr. Yu also serves as an Active Reviewer for Applied Physics Letters, Nanotechnology, Journal of Physics D: Applied Physics, Journal of the Electrochemical Society, and Electrochemical Solid-State Letters. He was a recipient of the Stanford Graduate Fellowship in 2009-2012 and the IEEE Electron Devices Society Masters Student Fellowship in 2010.

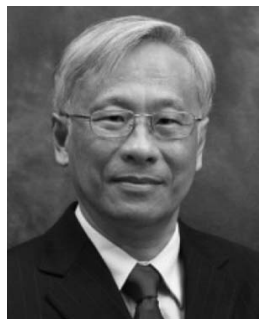

Philip C. H. Chan (SM'97-F'07) received the B.S. degree in electrical engineering from the University of California, Davis, in 1973 and the M.S. and $\mathrm{Ph} . \mathrm{D}$. degrees in electrical engineering from the University of Illinois at Urbana-Champaign (UIUC), Champaign, in 1975 and 1978, respectively.

He started his career as a Postdoctoral Fellow and an Assistant Professor with the UIUC. He later joined Intel Corporation in the U.S. and was promoted as a Senior Project Manager. In 1991, he was a Reader and a Founding Member of The Hong Kong University of Science and Technology (HKUST), Kowloon, Hong Kong, where he became the Chair Professor of the Department of Electronic and Computer Engineering. Since September 2003, he has been the Dean of Engineering of HKUST. He has also served as the Director of the Microelectronics Fabrication Facility, HKUST, and has received a total research funding of HK $\$ 71.7$ million since he joined the university. He has also been a key player in commercialization of the HKUST knowledge and technology. He is currently the Deputy President and Provost of The Hong Kong Polytechnic University, Kowloon, Hong Kong. His research interests include very large scale integration devices, circuits and systems, microelectronics, electronic packaging, integrated sensors, semiconductor device, and material research.

Prof. Chan is a Fellow of Hong Kong Institution of Engineering (HKIE). In Hong Kong, he has served in various innovation and technology commissions and HKIE committees, as well as the Electronics Committee of the Industry and Trade Department Council. He has advised the government on the setup of Hong Kong Applied Science and Technology Research Institute Company Ltd., where he currently serves in its board and is the Chair of the Technology Committee. He has also served as a Panel Member on the Research Grant Council and the University Grants Committee's Research Assessment Panel. He has an extensive network in the U.S. and has facilitated the collaboration between the HKUST and many topnotch engineering schools in the U.S., including the University of Pennsylvania, Cornell University, and the University of Southern California. He also holds honorary positions in various universities in Mainland China. He is the recipient of the Electrical and Computer Engineering Distinguished Alumni Award from the UIUC in 2010. 


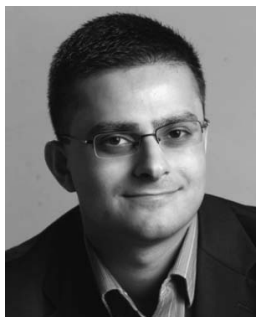

Ali Javey received the Ph.D. degree in chemistry from Stanford University, Stanford, CA, in 2005.

He served as a Junior Fellow of the prestigious Harvard Society of Fellows from 2005 to 2006. After being a Junior Fellow, he joined the faculty of the University of California, Berkeley, where he is currently an Associate Professor of electrical engineering and computer sciences. He is a Co-Director of the Berkeley Sensor and Actuator Center. His work focuses on the integration of nanoscale electronic materials for various technological applications, including novel nanoelectronics, flexible circuits and sensors, and energy generation and harvesting. His research interests encompass the fields of chemistry, materials science, and electrical engineering.

Assoc. Prof. Javey also serves as an Associate Editor of ACS Nano. For his contributions to the field, he became a recipient of a number of awards, including the IEEE Nanotechnology Early Career Award (2010), the Alfred P. Sloan Fellow (2010), the Mohr Davidow Ventures Innovators Award (2010), the National Academy of Sciences Award for Initiatives in Research (2009), the Technology Review TR35 (2009), the NSF Early CAREER Award (2008), the U.S. Frontiers of Engineering by National Academy of Engineering (2008), and the Peter Verhofstadt Fellowship from Semiconductor Research Corporation (2003).

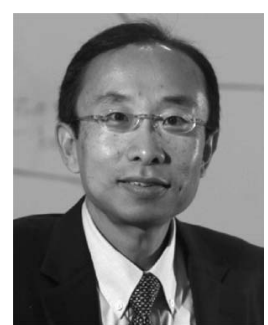

H.-S. Philip Wong (S'81-M'82-SM'95-F'01) received the B.Sc. degree (Honors) in electrical engineering from the University of Hong Kong, Kowloon, Hong Kong, in 1982, the M.S. degree in electrical engineering from the Stony Brook University-State University of New York, Stony Brook, in 1983, and the Ph.D. degree in electrical engineering from Lehigh University, Bethlehem, PA in 1988 .

He joined the IBM T. J. Watson Research Center, Yorktown Heights, NY, in 1988. In September 2004, he joined Stanford University, Stanford, CA, as a Professor of electrical engineering. While at IBM, he worked on charge-coupled device and complementary metal-oxide-semiconductor image sensors, double-gate/multigate MOSFET, device simulations for advanced/novel MOSFET, strained silicon, wafer bonding, ultrathin body silicon on insulator, extremely short gate FET, germanium MOSFET, carbon nanotube FET, and phase-change memory. $\mathrm{He}$ held various positions from being a Research Staff Member to Manager, and then Senior Manager. While he was a Senior Manager, he had the responsibility of shaping and executing IBM's strategy on nanoscale science and technology, as well as exploratory silicon devices and semiconductor technology. His research interests are in nanoscale science and technology, semiconductor technology, solid-state devices, and electronic imaging. He is interested in exploring new materials, novel fabrication techniques, and novel device concepts for future nanoelectronics systems. Novel devices often enable new concepts in circuit and system designs. His research also includes explorations into circuits and systems that are device driven. His current research covers a broad range of topics, including carbon nanotubes, self-assembly exploratory logic devices, nanoelectromechanical devices, novel memory devices, and biosensors.

He served in the IEEE Electron Devices Society as an elected Administrative Committee Member from 2001 to 2006. He served in the IEDM Committee from 1998 to 2007 and was the Technical Program Chair in 2006 and General Chair in 2007. He served in the ISSCC Program Committee from 1998 to 2004 and was the Chair of the Image Sensors, Displays, and Microelectromechanical system Subcommittee from 2003 to 2004. He serves in the Executive Committee of Symposia of VLSI Technology and Circuits. He was the Editor-inChief of the IEEE TRANSACTIONS ON NANOTECHNOLOGY in 2005-2006. He has been a Distinguished Lecturer of the IEEE Electron Devices Society (since 1999) and the Solid-State Circuit Society (2005-2007). 